\section{A Cooperative Sensing Based Cognitive Relay Transmission Scheme Without a Dedicated Sensing Relay Channel in Cognitive Radio Networks}

\author{
Yulong Zou, Yu-Dong Yao, and Baoyu Zheng
}

\begin{abstract}
In this correspondence, we investigate a selective relay spectrum sensing and best relay data transmission (SRSS-BRDT) scheme for multiple-relay cognitive radio networks. Specifically, in the spectrum sensing phase, only selected cognitive relays are utilized to transmit/forward their initial detection results (without a dedicated sensing relay channel) to a cognitive source for fusion, where the dedicated sensing channel refers to the channel transmitting initial spectrum sensing results from cognitive relays to the cognitive source. In the data transmission phase, only the best relay is selected to assist the cognitive source for its data transmissions. By jointly considering the two phases, we derive a closed-form expression of the outage probability for the SRSS-BRDT scheme over Rayleigh fading channels. We show that the SRSS-BRDT scheme outperforms the traditional cognitive transmission scheme (with a limited dedicated sensing channel) in terms of the outage probability performance. In addition, numerical results illustrate that the outage probability of the SRSS-BRDT scheme can be minimized through an optimal allocation of the time durations between the spectrum sensing and data transmission phases.
\end{abstract}

Index Terms-Cognitive radio, cognitive relay, cognitive transmission, cooperative sensing, outage probability.

\section{INTRODUCTION}

Cognitive radio is proposed as a means to improve the utilization of wireless spectrum resources, which enables unlicensed users to communicate with each other over licensed bands (through spectrum holes) [1], [2]. As discussed in [3]-[5], each cognitive transmission process requires two essential phases: 1) a spectrum sensing phase, in which a cognitive source attempts to detect an available spectrum hole; and 2) a data transmission phase, in which secondary data traffic (of the cognitive source) is transmitted to the destination through the detected spectrum hole. The two individual phases have been studied extensively in terms of different sensing [6]-[12] or different transmission [13]-[19] techniques.

However, as mentioned in [3]-[5], the spectrum sensing and data transmission phases can not be designed and optimized in isolation since the two phases affect each other. In [3], the authors focus on the maximization of secondary throughput under the constraint of primary user protection over additive white Gaussian noise (AWGN) channels. In [4], we have explored the sensing-and-transmission tradeoff issue over Rayleigh fading channels and shown that the outage probability of cog-

Manuscript received June 16, 2010; revised September 03, 2010; accepted October 24, 2010. Date of publication November 09, 2010; date of current version January 12, 2011. The associate editor coordinating the review of this manuscript and approving it for publication was Prof. Ye (Geoffrey) Li. This work was partially supported by the Postgraduate Innovation Program of Scientific Research of Jiangsu Province (Grant CX08B_080Z, CX09B_150Z) and the National Natural Science Foundation of China (Grant 60972039).

Y. Zou is with the Institute of Signal Processing and Transmission, Nanjing University of Posts and Telecommunications, Nanjing, Jiangsu 210003, China, and the Electrical and Computer Engineering Department, Stevens Institute of Technology, Hoboken, NJ 07030 USA (e-mail: zouyulong198412@126.com; yzou1@stevens.edu).

Y.-D. Yao is with the Electrical and Computer Engineering Department, Stevens Institute of Technology, Hoboken, NJ 07030, USA (e-mail: yyao@stevens.edu).

B. Zheng is with the Institute of Signal Processing and Transmission, Nanjing University of Posts and Telecommunications, Nanjing, Jiangsu 210003, China (e-mail: zby@njupt.edu.cn).

Color versions of one or more of the figures in this correspondence are available online at http://ieeexplore.ieee.org.

Digital Object Identifier 10.1109/TSP.2010.2090876 nitive transmissions can be minimized through the optimization of spectrum sensing overhead. Furthermore, we have investigated the cognitive transmissions with multiple relays in [5], where multiple cognitive relays are available to assist a cognitive source for both the spectrum sensing and data transmissions. In [5], we first propose a fixed fusion spectrum sensing and best relay data transmission (FFSS-BRDT) scheme and show that, as the number of cognitive relays increases, the performance of the FFSS-BRDT scheme improves initially and then begins to degrade when the number of cognitive relays is larger than a certain value. We then propose a selective fusion spectrum sensing and best relay data transmission (SFSS-BRDT) scheme, which performs better than FFSS-BRDT scheme. Moreover, the performance of SFSS-BRDT always improves as the number of cognitive relays increases.

Notice that both the FFSS-BRDT and SFSS-BRDT schemes employ the traditional cooperative sensing framework [8]-[11], where a dedicated channel is used when the cognitive relays forward their initial detection results to the cognitive source for fusion. This is somehow against the cognitive radio design principle, since cognitive radio is supposed to reuse the unoccupied licensed spectrum without dedicated channel (or, with very limited dedicated channel resources). Recently, in [12], we have proposed a selective-relay based cooperative sensing scheme, which can save the dedicated channel without receiver operating characteristics (ROC) performance degradation. In this correspondence, we consider the use of such a selective relay spectrum sensing scheme for cognitive transmissions to remove the dedicated sensing relay channel. The main contributions of this correspondence are described as follows. First, we propose a selective relay spectrum sensing and best relay data transmission (SRSS-BRDT) scheme, where only selected cognitive relays are utilized to transmit their initial detection results to a cognitive source for fusion and only the best relay is used to assist the cognitive source for its data transmissions. Secondly, jointly considering both the spectrum sensing and data transmission phases, we derive a closed-form expression of the outage probability for the SRSS-BRDT scheme. Finally, we show that the proposed SRSS-BRDT scheme can achieve a better outage probability performance, compared to the traditional SFSS-BRDT scheme with a limited dedicated channel resource.

The remainder of this correspondence is organized as follows. In Section II, we propose the SRSS-BRDT scheme for multiple-relay cognitive radio networks. Section III derives a closed-form expression of the outage probability for the proposed SRSS-BRDT scheme. Next, in Section IV, we conduct numerical outage probability evaluations for the SFSS-BRDT and SRSS-BRDT schemes. Finally, we provide concluding remarks in Section $\mathrm{V}$.

\section{PRoposed SRSS-BRDT SCHEME IN COGNITIVE RADIO NETWORKS}

\section{A. System Description}

As shown in Fig. 1, we consider a cognitive radio network, where multiple cognitive relays (CRs) are available to assist a cognitive source (CS) for both the spectrum sensing and data transmission phases. Following [13] and [14], a half-duplex relaying mode is adopted for CRs. Notice that there are $M$ CRs denoted by $\mathcal{R}=\left\{\mathrm{CR}_{i} \mid i=1,2, \ldots, M\right\}$. Fig. 2 shows the transmission protocol of the proposed selective relay spectrum sensing and best relay data transmission scheme. As seen from Fig. 2, each cognitive transmission process of the proposed SRSSBRDT scheme includes two phases (i.e., the spectrum sensing and data transmission phases), where the parameter $\alpha$ is referred to as spectrum sensing overhead, which can be adjusted to optimize the performance of cognitive transmissions.

Fig. 2 depicts that the spectrum sensing phase consists of two subphases. In the first subphase, CS and CRs independently detect the pres- 


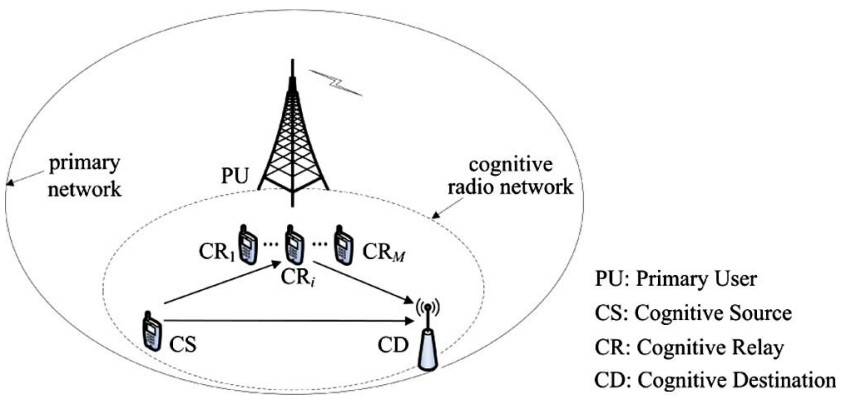

Fig. 1. Coexistence of a primary network and a cognitive radio network.

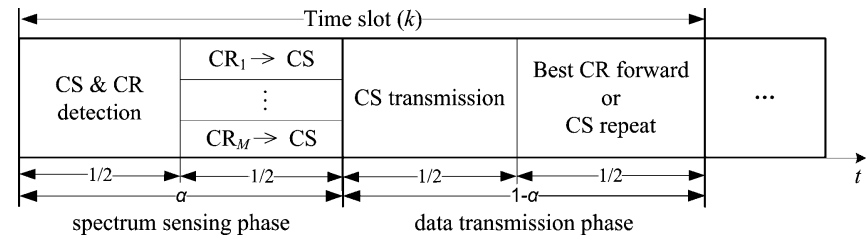

Fig. 2. Cognitive transmission protocol of the proposed selective relay spectrum sensing and best relay data transmission (SRSS-BRDT) scheme.

ence of a primary user (PU). Then, in the subsequent subphase, all CRs encode their initial detection results with an error detection code (such as, cyclic redundancy code), and transmit their encoded bits to CS over $M$ orthogonal primary licensed subchannels (instead of a dedicated channel), which will potentially interfere PU. In order to mitigate this interference, we consider the use of a selective relay spectrum sensing (SRSS) scheme [12], where each CRs forwards its initial detection result in a selective fashion. Specifically, if a CR detected the absence of PU in its detection phase, it will transmit a CRC-encoded indicator signal to CS; otherwise, nothing is transmitted from the CR to avoid interfering PU. Then, CS will perform CRC checking for the received signals from all the $M$ orthogonal subchannels. If the CRC checking is successful over $i$ th orthogonal subchannel, CS will consider the absence of $\mathrm{PU}$ as the initial result detected by $\mathrm{CR}_{i}$; otherwise, it will consider the presence of $\mathrm{PU}$ as the $\mathrm{CR}_{i}$ 's initial detection result. Accordingly, in the SRSS scheme, a CR will interfere the primary transmissions only if it fails to detect the presence of the primary user when $\mathrm{PU}$ is active. It has been proven in [12] that this interference is controllable and can be reduced to satisfy any given primary quality-of-service $(\mathrm{QoS})$ requirement.

In the data transmission phase, there are also two subphases. If a spectrum hole was detected earlier (in the sensing phase), CS will start transmitting its data to $\mathrm{CD}$ and CRs in the first data transmission subphase. Then, all CRs attempt to decode the CS' signal and those CRs which decode successfully constitute a set $D$, called a decoding set. Accordingly, the sample space of all the possible decoding sets is described as $\left\{\emptyset \cup D_{m}, m=1,2, \ldots 2^{M}-1\right\}$, where $\cup$ represents an union operation, $\emptyset$ is an empty set, and $D_{m}$ is a nonempty subcollection of the $M$ cognitive relays. In the second data transmission subphase, if the decoding set $(D)$ is not empty, the best relay (i.e., with the highest instantaneous signal-to-interference-and-noise ratio) chosen within the decoding set will forward its decoded result to $\mathrm{CD}$. If $D$ is empty, i.e., no relay is able to decode the CS' signal successfully, CS will repeat the transmission of the original signal to CD through its direct link. Finally, CD combines the two copies of received signals by using maximum ratio combining (MRC) method.

\section{B. Signal Modeling}

In the following, we formulate the signal model for the proposed SRSS-BRDT scheme. The transmit powers of the primary user and secondary users are denoted by $P_{p}$ and $P_{s}$, respectively. Let $H_{p}(k)$ represent, for time slot $k$, whether or not there is a spectrum hole. Specifically, $H_{p}(k)=H_{0}$ represents that a spectrum hole is available for secondary users; otherwise, $H_{p}(k)=H_{1}$. We model $H_{p}(k)$ as a Bernoulli random variable with parameter $\mathrm{P}_{\mathrm{a}}$ (the probability of the channel being available), i.e., $\operatorname{Pr}\left(H_{p}(k)=H_{0}\right)=\mathrm{P}_{\mathrm{a}}$ and $\operatorname{Pr}\left(H_{p}(k)=\right.$ $\left.H_{1}\right)=1-\mathrm{P}_{\mathrm{a}}$. In addition, the time-bandwidth product of the licensed channel is denoted by $B T$. In the first subphase of time slot $k$, the signal received at CS is expressed as

$$
y_{s}(k, 1)=\sqrt{P_{p}} h_{p s}(k) \theta(k, 1)+n_{s}(k, 1)
$$

where $h_{p s}(k)$ is the fading coefficient of the channel from PU to CS, $n_{s}(k, 1)$ is AWGN with zero mean and variance $N_{0}$, and $\theta(k, 1)$ is defined as

$$
\theta(k, 1)= \begin{cases}0, & H_{p}(k)=H_{0} \\ x_{p}(k, 1), & H_{p}(k)=H_{1}\end{cases}
$$

where $x_{p}(k, 1)$ is the transmit signal of PU in the first subphase of time slot $k$. Notice that $H_{p}(k)=H_{0}$ denotes that the channel is unoccupied by PU and nothing is transmitted from PU, and $H_{p}(k)=H_{1}$ represents that a PU signal is transmitted. Meanwhile, the signal received at $\mathrm{CR}_{i}$ is written as

$$
y_{i}(k, 1)=\sqrt{P_{p}} h_{p i}(k) \theta(k, 1)+n_{i}(k, 1), \quad i=1,2, \ldots, M
$$

where $h_{p i}(k)$ is the fading coefficient of the channel from PU to $\mathrm{CR}_{i}$ and $n_{i}(k, 1)$ is AWGN with zero mean and variance $N_{0}$. Based on the received signals as given by (1) and (2), CS and $\mathrm{CR}_{i}$ obtain their initial detection results, denoted by $\hat{H}_{s}(k, 1)$ and $\hat{H}_{i}(k, 1)$, respectively. Then, in the subsequent subphase, $\mathrm{CR}_{i}$ transmits a signal $\beta_{i}(k)$ over the corresponding orthogonal subchannel and the received signal at CS can be written as

$$
\begin{aligned}
y_{s}^{i}(k, 2)= & \sqrt{P_{s}} h_{i s}(k) \beta_{i}(k) \\
& +\sqrt{P_{p}} h_{p s}(k) \theta(k, 2)+n_{s}^{i}(k, 2), \quad i=1,2, \ldots, M
\end{aligned}
$$

where $h_{i s}(k)$ and $h_{p s}(k)$ are, respectively, the fading coefficients of the channel from $\mathrm{CR}_{i}$ to $\mathrm{CS}$ and that from PU to CS, and $\beta_{i}(k)$ and $\theta(k, 2)$ are defined as

$$
\beta_{i}(k)= \begin{cases}x_{i}(k), & \hat{H}_{i}(k, 1)=H_{0} \\ 0, & \hat{H}_{i}(k, 1)=H_{1}\end{cases}
$$

where $x_{i}(k)$ is an indicator signal that is encoded by a CRC code, and

$$
\theta(k, 2)= \begin{cases}0, & H_{p}(k)=H_{0} \\ x_{p}(k, 2), & H_{p}(k)=H_{1}\end{cases}
$$

where $x_{p}(k, 2)$ is the transmit signal of PU in the second subphase of time slot $k$. From (3), CS attempts to decode the signal $\beta_{i}(k)$ and perform CRC checking. As known in [13]-[15], if the channel capacity is below a required data rate, an outage event is said to occur and the decoder fails to recovery the original signal no matter what decoding algorithm is adopted. In this case, the CRC checking is assumed to fail and CS will consider that no indicator signal is transmitted from $\mathrm{CR}_{i}$, i.e., the corresponding initial detection result received at CS from $\mathrm{CR}_{i}$ is given by $\hat{H}_{i}(k, 2)=H_{1}$; otherwise, $\hat{H}_{i}(k, 2)=H_{0}$. Accordingly, we obtain

$$
\hat{H}_{i}(k, 2)= \begin{cases}H_{1}, & \Theta_{i s}(k, 2)=1 \\ H_{0}, & \Theta_{i s}(k, 2)=0\end{cases}
$$

where $\Theta_{i s}(k, 2)=1$ denotes that an outage event occurs over the channel from $\mathrm{CR}_{i}$ to $\mathrm{CS}$ and $\Theta_{i s}(k, 2)=0$ represents the other case. In 
an information-theoretic sense [12]-[15], the outage event $\Theta_{i s}(k, 2)=$ 1 can be described from (3) as

$\Theta_{i s}(k, 2)=1: \frac{\alpha}{2 M} \log _{2}\left(1+\frac{\left|h_{i s}(k)\right|^{2} \gamma_{s}\left|\beta_{i}(k)\right|^{2}}{\left|h_{p s}(k)\right|^{2} \gamma_{p}|\theta(k, 2)|^{2}+1}\right)<\frac{1}{B T}$

where $\gamma_{s}=P_{s} / N_{0}, \gamma_{p}=P_{p} / N_{0}$, and $B T$ is the time-bandwidth product of the licensed channel. Finally, CS combines all $\hat{H}_{i}(k, 2)$ and its own initial detection result $\hat{H}_{i}(k, 1)$ through a given fusion rule, leading to its final decision, $\hat{H}_{s}(k)$. Considering an "AND" rule, the final decision $\hat{H}_{s}(k)$ can be expressed as

$$
\hat{H}_{s}(k)=\hat{H}_{i}(k, 1) \underset{i=1}{\otimes} \hat{H}_{i}(k, 2)
$$

where $\otimes$ represents the logic AND operation. Next, we focus on the signal modeling for the data transmission phase. In the first part of the data transmission, i.e., the third subphase of time slot $k$, the signal received at $\mathrm{CD}$ is expressed as

$y_{d}(k, 3)=\sqrt{P_{s}} h_{s d}(k) \beta(k, 3)+\sqrt{P_{p}} h_{p d}(k) \theta(k, 3)+n_{d}(k, 3)$

where $h_{s d}(k)$ and $h_{p d}(k)$ are the fading coefficients of the channel from CS to $\mathrm{CD}$ and that from PU to $\mathrm{CD}$, respectively, and the parameters $\beta(k, 3)$ and $\theta(k, 3)$ are defined as

$$
\begin{aligned}
& \beta(k, 3)= \begin{cases}x_{s}(k), & \hat{H}_{s}(k)=H_{0} \\
0, & \hat{H}_{s}(k)=H_{1}\end{cases} \\
& \theta(k, 3)= \begin{cases}0, & H_{p}(k)=H_{0} \\
x_{p}(k, 3), & H_{p}(k)=H_{1}\end{cases}
\end{aligned}
$$

where $x_{s}(k)$ and $x_{p}(k, 3)$ are the transmit signals of CS and PU, respectively. Meanwhile, the signal received at $\mathrm{CR}_{i}$ can be written as

$$
\begin{aligned}
y_{i}(k, 3)= & \sqrt{P_{s}} h_{s i}(k) \beta(k, 3) \\
& +\sqrt{P_{p}} h_{p i}(k) \theta(k, 3)+n_{i}(k, 3), \quad i=1,2, \ldots, M
\end{aligned}
$$

where $h_{s i}(k)$ and $h_{p i}(k)$ are the fading coefficients of the channel from $\mathrm{CS}$ to $\mathrm{CR}_{i}$ and that from $\mathrm{PU}$ to $\mathrm{CR}_{i}$, respectively. In the fourth subphase, there are two possible cases for the data transmission depending on whether or not the decoding set $(D)$ is empty. For simplicity, let $D=\emptyset$ represent the first case of an empty decoding set and $D=D_{m}$ correspond to the other case, where $D_{m}$ is a nonempty subcollection set of all CRs.

- Case $D=\emptyset$ : This case corresponds to the scenario where all CRs fail to decode the signal from CS, implying

$$
\begin{aligned}
& \frac{(1-\alpha)}{2} \log _{2}\left(1+\frac{\left|h_{s i}(k)\right|^{2} \gamma_{s}|\beta(k, 3)|^{2}}{\left|h_{p i}(k)\right|^{2} \gamma_{p}|\theta(k, 3)|^{2}+1}\right) \\
& <R_{s}, \quad i=1,2, \ldots, M
\end{aligned}
$$

where $R_{s}$ is the data transmission rate of CS. In the given case $D=\emptyset$, CS will determine whether or not to repeat the transmission of signal $x_{s}(k)$ to $\mathrm{CD}$ depending on its final spectrum sensing result $\hat{H}_{s}(k)$, and thus the received signal at $\mathrm{CD}$ is given by

$$
\begin{aligned}
y_{d}(k, 4 \mid D=\emptyset)=\sqrt{P_{s}} h_{s d}(k) \beta(k, 4) \\
+\sqrt{P_{p}} h_{p d}(k) \theta(k, 4)+n_{d}(k, 4)
\end{aligned}
$$

where

$$
\begin{aligned}
& \beta(k, 4)= \begin{cases}x_{s}(k), & \hat{H}_{s}(k)=H_{0} \\
0, & \hat{H}_{s}(k)=H_{1}\end{cases} \\
& \theta(k, 4)= \begin{cases}0, & H_{p}(k)=H_{0} \\
x_{p}(k, 4), & H_{p}(k)=H_{1} .\end{cases}
\end{aligned}
$$

By combining (7) and (10) with the MRC method, CD can achieve an enhanced signal version with a signal-to-interference-and-noise ratio (SINR) as

$$
\begin{aligned}
& \operatorname{SINR}_{\mathrm{d}}(D=\emptyset) \\
& =\frac{\left|h_{s d}(k)\right|^{2} \gamma_{s}|\beta(k, 3)|^{2}+\left|h_{s d}(k)\right|^{2} \gamma_{s}|\beta(k, 4)|^{2}}{\left|h_{p d}(k)\right|^{2} \gamma_{p}|\theta(k, 3)|^{2}+\left|h_{p d}(k)\right|^{2} \gamma_{p}|\theta(k, 4)|^{2}+2} .
\end{aligned}
$$

- Case $D=D_{m}$ : This case corresponds to the scenario where CRs in decoding set $D_{m}$ are able to decode CS' signal successfully, i.e.,

$\frac{(1-\alpha)}{2} \log _{2}\left(1+\frac{\left|h_{s i}(k)\right|^{2} \gamma_{s}|\beta(k, 3)|^{2}}{\left|h_{p i}(k)\right|^{2} \gamma_{p}|\theta(k, 3)|^{2}+1}\right)>R_{s}, \quad i \in D_{m}$
$\frac{(1-\alpha)}{2} \log _{2}\left(1+\frac{\left|h_{s j}(k)\right|^{2} \gamma_{s}|\beta(k, 3)|^{2}}{\left|h_{p j}(k)\right|^{2} \gamma_{p}|\theta(k, 3)|^{2}+1}\right)<R_{s}, \quad j \in \bar{D}_{m}$ where $\bar{D}_{m}=\mathcal{R}-D_{m}$ is the complementary set of $D_{m}$. In this case, the cognitive relay, which can successfully decode the CS' signal and can achieve the highest received SINR at CD, is viewed as the "best" one and selected to forward the CS' signal to CD. Therefore, in the given case $D=D_{m}$, the combined SINR at CD is given by

$$
\begin{aligned}
& \operatorname{SINR}_{\mathrm{d}}\left(D=D_{m}\right) \\
& =\max _{i \in D_{m}} \frac{\left|h_{s d}(k)\right|^{2} \gamma_{s}+\left|h_{i d}(k)\right|^{2} \gamma_{s}}{\left|h_{p d}(k)\right|^{2} \gamma_{p}|\theta(k, 3)|^{2}+\left|h_{p d}(k)\right|^{2} \gamma_{p}|\theta(k, 4)|^{2}+2}
\end{aligned}
$$

where $D_{m}$ is the decoding set. One can observe from (13) that the best cognitive relay selection criterion takes into account the channel state information $\left|h_{s d}(k)\right|^{2},\left|h_{i d}(k)\right|^{2}$ and $\left|h_{p d}(k)\right|^{2}$. Using (13), we can further develop a specific relay selection algorithm in a centralized or distributed manner [17]-[20]. To realize the best cognitive relay selection, we can utilize a fraction of the detected spectrum holes of a licensed primary channel, instead of a dedicated control channel, for coordinating the different cognitive relays. Note that, if no spectrum hole is found, we do not need a dedicated channel for the best relay selection algorithm, since the cognitive source will not start transmitting data traffic in this case.

\section{OUtAGE ANALYSIS OF THE SRSS-BRDT SCHEME OVER RAYLEIGH FADING CHANNELS}

In this section, we derive a closed-form outage probability expression for the SRSS-BRDT scheme over Rayleigh fading channels. Following [13]-[15], an outage event is considered to occur when channel capacity falls below a predefined data transmission rate $R$. Accordingly, the outage probability of the proposed SRSS-BRDT scheme is calculated as

$$
\begin{aligned}
\text { Pout }= & \operatorname{Pr}\left\{\frac{1-\alpha}{2} \log _{2}\left(1+\operatorname{SINR}_{\mathrm{d}}\right)<R_{s}\right\} \\
= & \operatorname{Pr}\left\{\operatorname{SINR}_{\mathrm{d}}(D=\emptyset)<\gamma_{s} \Delta, D=\emptyset\right\} \\
& +\sum_{m=1}^{2^{M}-1} \operatorname{Pr}\left\{\operatorname{SINR}_{\mathrm{d}}\left(D=D_{m}\right)<\gamma_{s} \Delta, D=D_{m}\right\}
\end{aligned}
$$

where $\Delta=\left[2^{2 R_{s} /(1-\alpha)}-1\right] / \gamma_{s}, \operatorname{SINR}_{\mathrm{d}}(D=\emptyset)$ and $\operatorname{SINR}_{\mathrm{d}}(D=$ $\left.D_{m}\right)$ are given by (11) and (13), respectively. According to (9) and (11), the term $\operatorname{Pr}\left\{\operatorname{SINR}_{\mathrm{d}}(D=\emptyset)<\gamma_{s} \Delta, D=\emptyset\right\}$ in the second equation of (14) can be expanded as

$$
\begin{aligned}
\operatorname{Pr}\left\{\operatorname{SINR}_{\mathrm{d}}(D=\emptyset)<\gamma_{s} \Delta, D=\emptyset\right\} \\
=\mathrm{P}_{\mathrm{a}}\left(1-\operatorname{Pf}_{s}\right) \operatorname{Pr}\left\{\left|h_{s d}(k)\right|^{2}<\Delta\right\} \\
\quad \times \prod_{i=1}^{M} \operatorname{Pr}\left\{\left|h_{s i}(k)\right|^{2}<\Delta\right\}\left(1-\mathrm{P}_{\mathrm{a}}\right)\left(1-\operatorname{Pd}_{s}\right) \\
\quad \times \operatorname{Pr}\left\{\left|h_{s d}(k)\right|^{2}-\left|h_{p d}(k)\right|^{2} \gamma_{p} \Delta<\Delta\right\} \\
\quad \times \prod_{i=1}^{M} \operatorname{Pr}\left\{\left|h_{s i}(k)\right|^{2}-\left|h_{p i}(k)\right|^{2} \gamma_{p} \Delta<\Delta\right\} \\
+\mathrm{P}_{\mathrm{a}} \operatorname{Pf}_{s}+\left(1-\mathrm{P}_{\mathrm{a}}\right) \operatorname{Pd}_{s}
\end{aligned}
$$

where $\mathrm{P}_{\mathrm{a}}=\operatorname{Pr}\left\{H_{p}(k)=H_{0}\right\}$ is the probability that there is a spectrum hole, $\operatorname{Pd}_{s}=\operatorname{Pr}\left\{\hat{H}_{s}(k)=H_{1} \mid H_{p}(k)=H_{1}\right\}$ and $\operatorname{Pf}_{s}=$ $\operatorname{Pr}\left\{\hat{H}_{s}(k)=H_{1} \mid H_{p}(k)=H_{0}\right\}$ are, respectively, the probabilities of overall detection and false alarm of the PU's presence at CS after final fusion, as shown in (6). Besides, the probabilities in (15) (e.g., 
$\operatorname{Pr}\left\{\left|h_{s i}(k)\right|^{2}<\Delta\right\}, \operatorname{Pr}\left\{\left|h_{s d}(k)\right|^{2}-\left|h_{p d}(k)\right|^{2} \gamma_{p} \Delta<\Delta\right\}$, and so on) can be easily calculated with closed-form solutions, since random variables $\left|h_{s d}(k)\right|^{2},\left|h_{p d}(k)\right|^{2},\left|h_{s i}(k)\right|^{2}$ and $\left|h_{p i}(k)\right|^{2}$ follow exponential distributions and are independent from each other. From (12) and (13), the term $\operatorname{Pr}\left\{\operatorname{SINR}_{\mathrm{d}}\left(D=D_{m}\right)<\gamma_{s} \Delta, D=D_{m}\right\}$ in the second equation of (14) is found as

$$
\begin{aligned}
& \operatorname{Pr}\left\{\operatorname{SINR}_{\mathrm{d}}\left(D=D_{m}\right)<\gamma_{s} \Delta, D=D_{m}\right\} \\
&=\operatorname{Pa}_{\mathrm{a}}\left(1-\operatorname{Pf}_{s}\right) \\
& \quad \times \operatorname{Pr}\left\{\max _{i \in D_{m}}\left|h_{i d}(k)\right|^{2}<2 \Delta-\left|h_{s d}(k)\right|^{2}\right\} \\
& \quad \times \prod_{i \in D_{m}} \operatorname{Pr}\left\{\left|h_{s i}(k)\right|^{2}>\Delta\right\} \\
& \quad \times \prod_{j \in \bar{D}_{m}} \operatorname{Pr}\left\{\left|h_{s j}(k)\right|^{2}<\Delta\right\} \\
& \quad+\left(1-\operatorname{Pa}_{\mathrm{a}}\right)\left(1-\operatorname{Pd}_{s}\right) \operatorname{Pr}\left\{\max _{i \in D_{m}}\left|h_{i d}(k)\right|^{2}\right. \\
&<\left.2 \Delta-\left|h_{s d}(k)\right|^{2}+2\left|h_{p d}(k)\right|^{2} \gamma_{p} \Delta\right\} \\
& \times \prod_{i \in D_{m}} \operatorname{Pr}\left\{\left|h_{s i}(k)\right|^{2}-\left|h_{p i}(k)\right|^{2} \gamma_{p} \Delta>\Delta\right\} \\
& \times \prod_{j \in \bar{D}_{m}} \operatorname{Pr}\left\{\left|h_{s j}(k)\right|^{2}-\left|h_{p j}(k)\right|^{2} \gamma_{p} \Delta<\Delta\right\}
\end{aligned}
$$

where the closed-form solution to $\operatorname{Pr}\left\{\max _{i \in D_{m}}\left|h_{i d}(k)\right|^{2}<2 \Delta-\right.$ $\left.\left|h_{s d}(k)\right|^{2}+2\left|h_{p d}(k)\right|^{2} \gamma_{p} \Delta\right\}$ has been derived as given by [20, eq. (24)]. Now, we start the analysis of the probabilities of overall detection and false alarm of the PU's presence at CS, i.e., the terms $\mathrm{Pd}_{s}$ and $\mathrm{Pf}_{s}$ as given in (15) and (16). Using (4)-(6) and following [12], the overall detection probability $\mathrm{Pd}_{s}$ for the selective relay spectrum sensing is calculated as

$$
\operatorname{Pd}_{s}=\operatorname{Pd}_{s, 1} \prod_{i=1}^{M}\left[1-\frac{\sigma_{i s}^{2}\left(1-\operatorname{Pd}_{i, 1}\right)}{\sigma_{p s}^{2} \gamma_{p} \Lambda+\sigma_{i s}^{2}} \exp \left(-\frac{\Lambda}{\sigma_{i s}^{2}}\right)\right]
$$

where $\sigma_{i s}^{2}=E\left[\left|h_{i s}(k)\right|^{2}\right], \sigma_{p d}^{2}=E\left[\left|h_{p d}(k)\right|^{2}\right], \Lambda=\left[2^{2 M /(\alpha B T)}-\right.$ $1] / \gamma_{s}, \operatorname{Pd}_{s, 1}=\operatorname{Pr}\left\{\hat{H}_{s}(k, 1)=H_{1} \mid H_{p}(k)=H_{1}\right\}$ and $\operatorname{Pd}_{i, 1}=$ $\operatorname{Pr}\left\{\hat{H}_{i}(k, 1)=H_{1} \mid H_{p}(k)=H_{1}\right\}$ are the probabilities of individual detection of the PU's presence at CS and $\mathrm{CR}_{i}$, respectively. Similarly, the false detection probability $\operatorname{Pf}_{s}$ is given by

$$
\operatorname{Pf}_{s}=\operatorname{Pf}_{s, 1} \prod_{i=1}^{M}\left[1-\left(1-\operatorname{Pf}_{i, 1}\right) \exp \left(-\frac{\Lambda}{\sigma_{i s}^{2}}\right)\right]
$$

where $\operatorname{Pf}_{s, 1}=\operatorname{Pr}\left\{\hat{H}_{s}(k, 1)=H_{1} \mid H_{p}(k)=H_{0}\right\}$ and $\operatorname{Pf}_{i, 1}=\operatorname{Pr}\left\{\hat{H}_{i}(k, 1)=H_{1} \mid H_{p}(k)=H_{0}\right\}$ are the probabilities of individual false alarm of the PU's presence at $\mathrm{CS}$ and $\mathrm{CR}_{i}$, respectively. Considering an energy detector, $\mathrm{Pd}_{s, 1}$ and $\mathrm{Pf}_{s, 1}$ can be calculated as $\operatorname{Pd}_{s, 1}=\operatorname{Pr}\left\{T\left[y_{s}(k, 1)\right]>\delta \mid H_{p}(k)=H_{1}\right\}$ and $\operatorname{Pf}_{s, 1}=\operatorname{Pr}\left\{T\left[y_{s}(k, 1)\right]>\delta \mid H_{p}(k)=H_{0}\right\}$, where $\delta$ is an energy detection threshold and $T\left[y_{s}(k, 1)\right]$ is an output statistic of the energy detector as given by

$$
T\left[y_{s}(k, 1)\right]=\frac{1}{N} \sum_{n=1}^{N}\left|y_{s}^{n}(k, 1)\right|^{2}
$$

where $N$ is the number of samples. Using the results of [12, App. A], we can obtain

$$
\operatorname{Pf}_{s, 1}=\left\{\begin{array}{l}
\operatorname{Pd}_{s, 1}, \quad \operatorname{Pd}_{s, 1}=Q(-\sqrt{N}) \\
\operatorname{Pd}_{s, 1}-Q\left(Q^{-1}\left(\operatorname{Pd}_{s, 1}\right)+\frac{1}{\sigma_{p s}^{2} \kappa_{s}}\right) \exp \left(\xi_{s}\right), \\
\text { otherwise }
\end{array}\right.
$$

where $\kappa_{s}=\gamma_{p} Q^{-1}\left(\operatorname{Pd}_{s, 1}\right)+\sqrt{N} \gamma_{p}, \xi_{s}=Q^{-1}\left(\operatorname{Pd}_{s, 1}\right) / \sigma_{p s}^{2} \kappa_{s}+$ $1 / 2 \sigma_{p s}^{4} \kappa_{s}^{2}$, and the number of samples should satisfy $N \geq$ $\left[Q^{-1}\left(\operatorname{Pd}_{s, 1}\right)\right]^{2}$. Similar to the derivation of (20) and following (2), we obtain

$$
\operatorname{Pf}_{i, 1}=\left\{\begin{array}{l}
\mathrm{Pd}_{i, 1}, \\
\mathrm{Pd}_{i, 1}-Q\left(Q^{-1}\left(\mathrm{Pd}_{i, 1}\right)+\frac{1}{\sigma_{p i}^{2} \kappa_{i}}\right) \exp \left(\xi_{i}\right), \\
\text { otherwise }
\end{array}\right.
$$

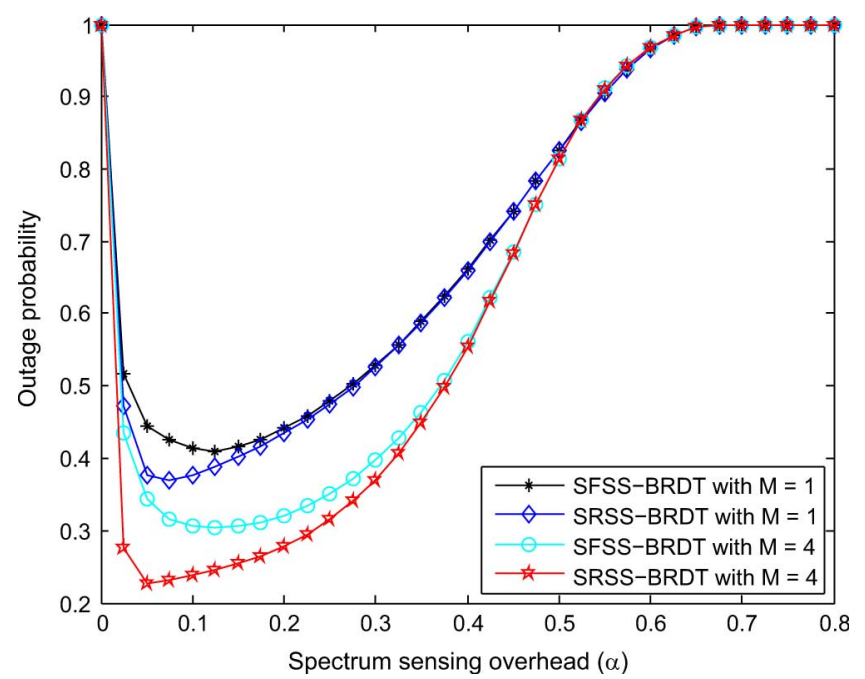

Fig. 3. Outage probability versus the spectrum sensing overhead $\alpha$ of the traditional SFSS-BRDT and proposed SRSS-BRDT schemes for different number of CRs $M$ with $\mathrm{P}_{\mathrm{a}}=0.8, \mathrm{Pd}_{s}=0.99, \gamma_{p}=10 \mathrm{~dB}, \gamma_{s}=10 \mathrm{~dB}, R_{s}=$ $1 \mathrm{~b} / \mathrm{s} / \mathrm{Hz}, B T=500, B_{d} T_{d}=50, f_{s}=50 \mathrm{kHz}, R_{p}=2 \mathrm{~b} / \mathrm{s} / \mathrm{Hz}$, and Pout $_{\text {pri,thr }}=10^{-3}$, where $R_{p}$ and Pout pri,thr $_{\text {are the primary data rate and }}$ primary outage probability requirement, respectively.

where $\kappa_{i}=\gamma_{p} Q^{-1}\left(\operatorname{Pd}_{i, 1}\right)+\sqrt{N} \gamma_{p}, \xi_{i}=Q^{-1}\left(\operatorname{Pd}_{i, 1}\right) / \sigma_{p i}^{2} \kappa_{i}+$ $1 / 2 \sigma_{p i}^{4} \kappa_{i}^{2}$, and $N \geq\left[Q^{-1}\left(\operatorname{Pd}_{i, 1}\right)\right]^{2}$. Note that, in the proposed SRSSBRDT scheme, a primary user may be interfered by the cognitive users during both the spectrum sensing and data transmission phases. Specifically, in the spectrum sensing phase, a cognitive relay will interfere the primary user if it fails to detect the presence of the primary user and, in the data transmission phase, the primary user will be interfered by the secondary transmissions when the cognitive source node made a miss detection of the PU's presence. Nevertheless, any given primary QoS requirement can be satisfied by adjusting the individual detection probability $\mathrm{Pd}_{i, 1}$ as given by [12, eq. (40)].

\section{NUMERICAL RESULTS AND ANALYSIS}

In this section, we conduct numerical outage probability evaluations for the traditional SFSS-BRDT scheme (with a dedicated channel) [5] and the proposed SRSS-BRDT scheme (without a dedicated channel). Notice that, in the following numerical evaluations, the fading variances of the channel between each sender-receiver within a same network (primary or secondary networks) and that across different networks are specified to 1 and 0.5 , respectively. Fig. 3 shows the outage probability versus spectrum sensing overhead of the traditional SFSSBRDT and proposed SRSS-BRDT schemes for different number of CRs, $M$, where the time-bandwidth products of the licensed primary channel and dedicated sensing channel are $B T=500$ and $B_{d} T_{d}=$ 50 , respectively. This considers that the cognitive radio is typically designed to reuse the licensed spectrum with very limited dedicated channel resources. As shown in Fig. 3, the outage probabilities of the proposed SRSS-BRDT scheme are lower than that of the traditional SFSS-BRDT scheme for $M=1$ and $M=4$, respectively. In addition, one can see from Fig. 3 that the outage probabilities of both the traditional and proposed schemes can be minimized through adjusting the spectrum sensing overhead. Therefore, a joint analysis of the spectrum sensing and data transmission phases is essential to optimize the cognitive transmission performance.

Fig. 4 illustrates the outage probability versus spectrum sensing overhead of the SFSS-BRDT and SRSS-BRDT schemes for different data transmission rates $R_{s}$. All cases in Fig. 4 show that the proposed SRSS-BRDT scheme outperforms the traditional SFSS-BRDT scheme in terms of the outage probability. From Fig. 4, one can also observe that an optimal spectrum sensing overhead exists to minimize the 


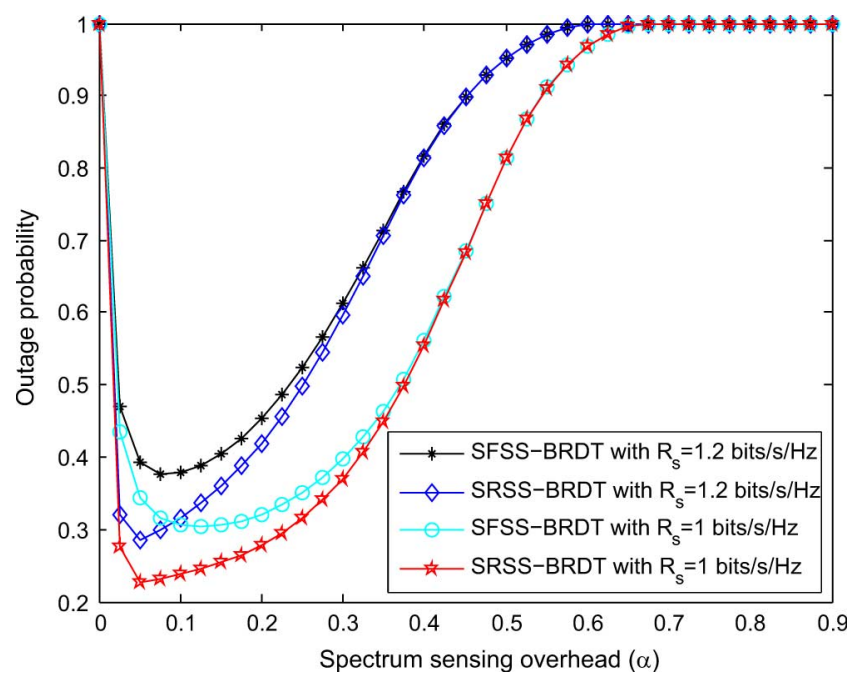

Fig. 4. Outage probability versus the spectrum sensing overhead $\alpha$ of the traditional SFSS-BRDT and proposed SRSS-BRDT schemes for different data transmission rates $R_{s}$ with $\mathrm{P}_{\mathrm{a}}=0.8, \mathrm{Pd}_{s}=0.99, \gamma_{p}=10 \mathrm{~dB}, \gamma_{s}=10 \mathrm{~dB}, R=$ $1 \mathrm{~b} / \mathrm{s} / \mathrm{Hz}, M=4, B T=500, B_{d} T_{d}=50, f_{s}=50 \mathrm{kHz}, R_{p}=2 \mathrm{~b} / \mathrm{s} / \mathrm{Hz}$, and Pout $\mathrm{pri,thr}=10^{-3}$.

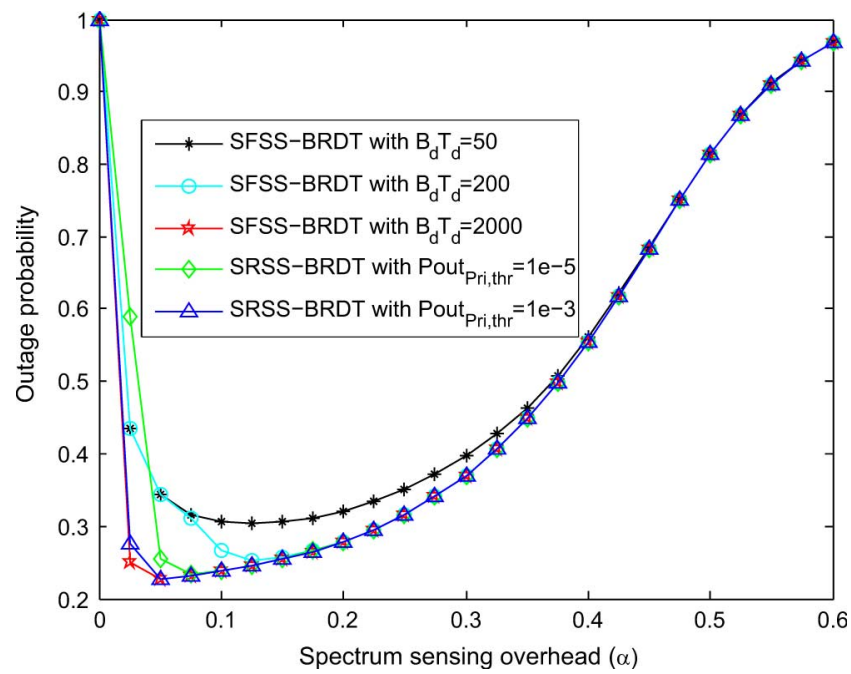

Fig. 5. Outage probability comparison between the traditional SFSS-BRDT scheme and proposed SRSS-BRDT scheme for different time-bandwidth products of the dedicated channel $B_{d} T_{d}$ with $\mathrm{P}_{\mathrm{a}}=0.8, \mathrm{Pd}_{s}=0.99, \gamma_{p}=10 \mathrm{~dB}$, $\gamma_{s}=10 \mathrm{~dB}, R_{s}=1 \mathrm{~b} / \mathrm{s} / \mathrm{Hz}, M=4, B T=500, f_{s}=50 \mathrm{kHz}$, and $R_{p}=2 \mathrm{~b} / \mathrm{s} / \mathrm{Hz}$.

outage probability and, moreover, the optimal spectrum sensing overhead value decreases gradually with an increasing data rate $R_{s}$. This is due to the fact that, as the data rate $R_{s}$ increases, the data transmission phase should be assigned a longer time duration, resulting in a shorter time duration for the spectrum sensing phase.

In Fig. 5, we show the outage probability comparison between the SFSS-BRDT scheme (with different time-bandwidth products of the dedicated channel $B_{d} T_{d}$ ) and SRSS-BRDT scheme (with different primary outage probability requirements Pout pri,thr ). As shown in Fig. 5, as the time-bandwidth product $B_{d} T_{d}$ increases from $B_{d} T_{d}=50$ to 2000 , the outage probability curves of the traditional SRSS-BRDT scheme become closer to that of the proposed SRSS-BRDT scheme with Pout $t_{\text {pri,thr }}=10^{-3}$. One can also see from Fig. 5 that, when the primary outage probability requirement is very stringent, i.e., Pout $_{\text {pri,thr }}=10^{-5}$, the SRSS-BRDT performs worse than the SFSS-BRDT in terms of the outage probability. However, notice that an overly strict primary outage probability requirement is not practical and thus the advantage of the SRSS-BRDT scheme is achievable in practical wireless transmission systems.

\section{CONCLUSION}

In this correspondence, we have investigated a selective relay spectrum sensing and best relay data transmission scheme for multiple-relay cognitive radio networks. We have derived a closed-form outage probability expression for the SRSS-BRDT scheme over Rayleigh fading channels. Numerical results have demonstrated that the SRSS-BRDT scheme can save the dedicated channel without outage probability degradation, compared to the SFSS-BRDT scheme with a limited dedicated channel resource. We have also shown that a minimum outage probability can be achieved through an optimal allocation of the time durations between the spectrum sensing and data transmission phases.

\section{REFERENCES}

[1] J. Mitola and G. Q. Maguire, "Cognitive radio: Making software radios more personal," IEEE Personal Commun., vol. 6, pp. 13-18, 1999.

[2] S. Haykin, "Cognitive radio: Brain-empowered wireless communications," IEEE J. Sel. Areas Commun., vol. 23, no. 2, pp. 201-220, 2005.

[3] Y.-C. Liang, Y. Zeng, E. Peh, and A. T. Hoang, "Sensing-throughput tradeoff for cognitive radio networks," IEEE Trans. Wireless Commun., vol. 7, no. 4, pp. 1326-1337, Apr. 2008.

[4] Y. Zou, Y.-D. Yao, and B. Zheng, "Outage probability analysis of cognitive transmissions: The impact of spectrum sensing overhead," IEEE Trans. Wireless Commun., vol. 9, no. 8, pp. 2676-2688, Aug. 2010.

[5] Y. Zou, Y.-D. Yao, and B. Zheng, "Cognitive transmissions with multiple relays in cognitive radio networks," IEEE Trans. Wireless Commun., vol. 10, 2011.

[6] V. I. Kostylev, "Energy detection of a signal with random amplitude," in Proc. IEEE Int. Conf. Commun., 2002, pp. 1606-1610.

[7] P. D. Sutton, K. E. Nolan, and L. E. Doyle, "Cyclostationary signatures in practical cognitive radio applications," IEEE J. Sel. Areas Commun., vol. 26, no. 1, pp. 13-24, 2008.

[8] A. Ghasemi and E. S. Sousa, "Collaborative spectrum sensing for opportunistic access in fading environment," in Proc. IEEE DySPAN 2005, pp. 131-136.

[9] G. Ganesan and Y. G. Li, "Cooperative spectrum sensing in cognitive radio, part I: Two user networks," IEEE Trans. Wireless Commun., vol. 6, no. 6, pp. 2204-2213, 2007.

[10] J. Ma, G. Zhao, and Y. Li, "Soft combination and detection for cooperative spectrum sensing in cognitive radio networks," IEEE Trans. Wireless Commun., vol. 7, no. 11, pp. 4502-4507, Nov. 2008.

[11] J. Zhu, Y. Zou, and B. Zheng, "Cooperative detection for primary user in cognitive radio networks," EURASIP J. Wireless Commun. and Networking, , 2009, DOI: 10.1155/2009/617320 [Online]. Available: http://downloads.hindawi.com/journals/wcn/2009/617320.pdf

[12] Y. Zou, Y.-D. Yao, and B. Zheng, "A selective-relay based cooperative spectrum sensing scheme without dedicated relay channels in cognitive radio networks," IEEE Trans. Wireless Commun., unpublished.

[13] J. N. Laneman, D. N. C. Tse, and G. W. Wornell, "Cooperative diversity in wireless networks: Efficient protocols and outage behavior," IEEE Trans. Inf. Theory, vol. 50, no. 12, pp. 3062-3080, 2004.

[14] T. E. Hunter, S. Sanayei, and A. Nosratinia, "Outage analysis of coded cooperation," IEEE Trans. Inf. Theory, vol. 52, no. 2, pp. 375-391, 2006.

[15] Y. Zou, B. Zheng, and J. Zhu, "Outage analysis of opportunistic cooperation over Rayleigh fading channels," IEEE Trans. Wireless Commun., vol. 8, no. 6, pp. 3077-3385, Jun. 2009.

[16] Y. Zou, B. Zheng, and W.-P. Zhu, "An opportunistic cooperation scheme and its ber analysis," IEEE Trans. Wireless Commun., vol. 8, no. 9, pp. 4492-4497, Sep. 2009.

[17] A. Bletsas, H. Shin, M. Z. Win, and A. Lippman, "A simple cooperative diversity method based on network path selection," IEEE J. Sel. Areas Commun., vol. 24, no. 3, pp. 659-672, Mar. 2006.

[18] E. Beres and R. S. Adve, "Selection cooperation in multi-source cooperative networks," IEEE Trans. Wireless Commun., vol. 7, no. 1, pp. 118-127, 2008.

[19] S. Lkki and M. H. Ahmed, "Performance of multiple-relay cooperative diversity systems with best relay selection over Rayleigh fading channels," EURASIP J. Adv. Signal Process., 2009.

[20] Y. Zou, J. Zhu, B. Zheng, and Y.-D. Yao, "An adaptive cooperation diversity scheme with best-relay selection in cognitive radio networks," IEEE Trans. Signal Process, vol. 58, no. 10, pp. 5438-5445, Oct. 2010. 